\title{
Preliminary Experience with Rifampicin and Isoprodian (L73A) - Combination in Lepromatous Leprosy
}

\author{
M. HAMZAH AND A. KOSASIH \\ Department of Dermatology, University of Indonesia, \\ Jakarta, Indonesia
}

\begin{abstract}
Nineteen male patients suffering from lepromatous leprosy are being treated with rifampicin and Isoprodian. So far, 8 patients have completed 6 months of follow-up, 5 patients have received treatment for less than 6 months and another 6 patients have just started treatment.

Out of 13 patients under treatment, 10 patients so far have become bacteriologically negative, accompanied by clinical improvement, especially in the macular, nodular and diffuse types of infiltration, whereas miliary and lenticular types of infiltration improved slightly.

Side-effects were mild, except in 1 patient who dropped out because of severe vomiting.

Laboratory examinations showed no significant changes in the blood picture and the initial high value of ESR gradually decreased.
\end{abstract}

\section{Introduction}

Those who have devoted a long time to the treatment of leprosy are aware of the inadequacies of present day therapy. Even the drug most widely applied at the present time, namely dapsone, is still under research to find out the most efficient dose requirements, and the search for a more efficient drug continues.

Experiments of various combinations of drugs have been made, both using known as well as combinations of completely new drugs in the treatment of leprosy. In the present experiment the therapy applied is a combination of drugs composed of rifampicin and Isoprodian (L73A). Rifampicin was applied for the first time in leprosy in 1965. Each tablet of Isoprodian used in this investigation contains a combination of $3 \mathrm{drugs}$, namely dapsone $50 \mathrm{mg}$, Isoniazid $250 \mathrm{mg}$ and $250 \mathrm{mg}$ Prothionamide.

Dapsone is the most widely applied drug, Isoniazid is not a drug to be applied routinely in leprosy.

Prothionamide is a drug applied for the first time in leprosy in Indonesia.

\section{Materials and Methods}

The experiment was carried out at the General Hospital in Jakarta (RSTM), subdivision for leprosy outpatient Department. 
It was intended to treat at least 20 patients, all male, and of the lepromatous type, with complete follow-up examination.

The reason why only male patients were chosen was the fact that thalidomide would be used in the event of lepra reaction. These patients were chosen carefully in order to minimize drop-outs, and considering the need for frequent follow-up examinations.

Each new patient was given the following examinations:

(1) $10 \mathrm{ml}$ of blood was drawn for examination of $\mathrm{Hb}$, leucocyte count, BSR, differential count, thrombocyte count, SGOT and SGPT

(2) A direct smear of the lesion for bacterioscopical examination

(3) A skin biopsy for histopathological examination

(4) A coloured photograph of the lesion

All these examinations were done at the same day whenever possible.

Follow-up examinations were undertaken monthly to evaluate the clinical progress, observe side-effects, and perform the same laboratory procedures as mentioned above. Treatment accompanied by 6 times follow-up would be completed in 6-7 months.

The treatment was started in September, 1973, by using rifampicin $2 \times 300 \mathrm{mg} /$ day and $3 \times 1$ tablet of L73A. Later on, treatment was modified to $2 \times 300 \mathrm{mg}$ rifampicin and 2 tablets of Isoprodian ( 1 tablet of Isoprodian is equal to 2 tablets of L73A used formerly) with 1 day pause within each week, namely on Sunday. This quantity of drug was given in a single dose with breakfast. However, when the single dose treatment was difficult, patients were allowed to take the drug in divided doses. This dose was given to patients whose body weight was more than $40 \mathrm{~kg}$, whereas patients weighing less than $40 \mathrm{~kg}$ were given half of the dose.

\section{Results}

This preliminary report relates to the results of the treatment of 13 male lepromatous patients at ages varying between 11 and 65 years.

Eight patients have completed the 6 times follow-up. Five patients are under treatment for the duration of less than 6 times follow-up, specified as follows:

Two patients have reached the 5 th follow-up, 2 patients have reached the 4 th follow-up and 1 patient has reached the 2 nd follow-up.

It is hoped that all these patients will manage to complete the treatment in due time. Another 6 patients have just started treatment. Besides these patients there were 6 patients who have stopped the treatment in the early phases of treatment for the following reasons:

Two patients rejected the second biopsy, 1 patient because of the occurrence of a big keloid on the biopsy scar, 1 patient because of severe vomiting, restlessness and insomnia, whereas 2 patients have not returned for unknown reasons.

\section{Clinical Results}

The skin lesions found were in the form of erythematous macules of numular size, usually spread over the trunk, sometimes on the arms and thighs, diffuse infiltration and erythematous nodules usually found on the face, ear, extremities 
and sometimes on the trunk; and smaller nodular lesions of miliary and lenticular size, usually found on the ear, and in 1 patient occurring on the lips, while in another patient found spread over the back and limbs.

During the first weeks of treatment, improvement of the lesions was readily seen, especially the nodules and diffuse infiltrations, whereas with nodular lesions of miliar and lenticular size very slight or no improvement was observed.

Macular lesions disappeared without leaving a mark. Diffuse infiltrated lesions and nodules partly disappeared without leaving a mark, partly leaving macules, partly leaving a wrinkled skin the colour of which is normal or bluish.

In addition, there was also infiltration which underwent only a slight change either in terms of thickness or colouring.

Relating to this improvement, there was a difference both individually and topographically. Relating to the changes in miliary and lenticular noduli, a slight improvement took place except in the erythema.

Infiltrates that took a long time to regress were those localized on the ear, face and the distal part of the extremities.

\section{Notes on patients of interest}

(1) A. L., age 65. After 6 follow-up examinations no trace of infiltration was to be seen on the skin, except that a few bullae were always found, appearing and vanishing, containing clear or haemorrhagic fluid mainly on the palm of the right hand, sometimes on the palm of the left hand and on the sole of the infected foot. This lesion occurred immediately before the treatment and during the treatment.

(2) M. M., age 31. After 6 follow-up examinations persistent lesions remained in the form of nodules of miliary size in both ears, infiltration of the lower right leg with edema.

Persistent painful enlargement of the right femoral gland occurred 3 months after the treatment. This patient appeared to suffer from active tuberculosis at the 5 th follow-up, with the occurrence of haemoptosis. A sputum direct smear examination showed a negative result, while the result of culture is still awaited.

(3) W. A., age 50. Before treatment there were lesions in the form of nodules spread over the whole body and the existence of facies leonina. During the treatment a few lesions changed to become erosive, excoriative and ulcerative after the 1st follow-up for a period of approximately 1 month at the 6th examination. Now, the lesions have become hyper-pigmented macules, wrinkled and encircled by small infiltrates which were somewhat ery thematous.

(4) M. R., age 37. At the sixth monthly examination, the noduli of miliar and lenticular size on the back, breast, upper arm and neck persist.

(5) Z. A., age 30. At the sixth monthly examination the lesions remained unchanged in the form of miliary nodules on the upper and lower lip and on the nose. Both ears were irregularly thickened with shiny face due to the presence of a diffuse infiltrate of bluish colour. The fingers as well as the skin covering the extensor part of the arm and the lower third of the leg were also shiny and of a bluish colour.

(6) A. G., age 42. Prior to this trial he was treated with Conteben for $1 \frac{1}{2}$ years, with Ciba 1906 during 1 1 $1 / 2$ years, with Lamprene for 15 months and with dapsone for 1 month, without any progress. After 5 months with this new method of treatment progress was quite satisfactory. It was seen objectively that the oedema on the lower leg disappeared entirely and there was improvement in all 


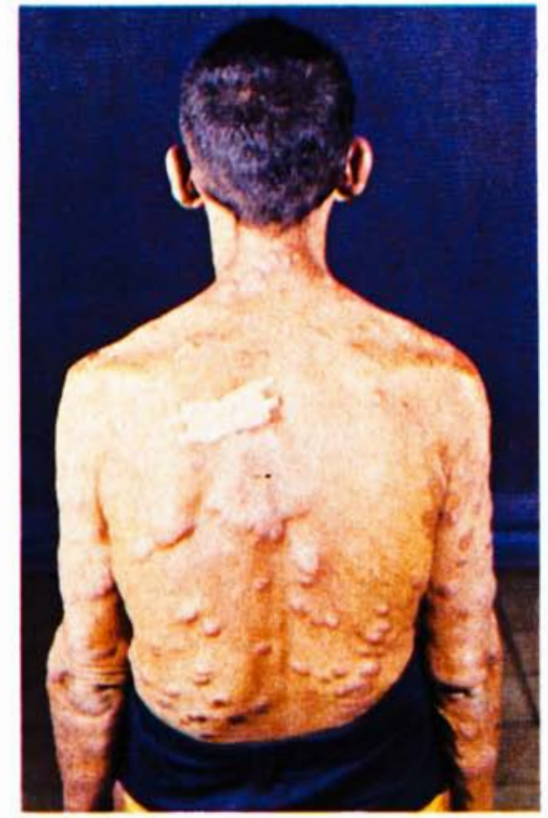

(a)

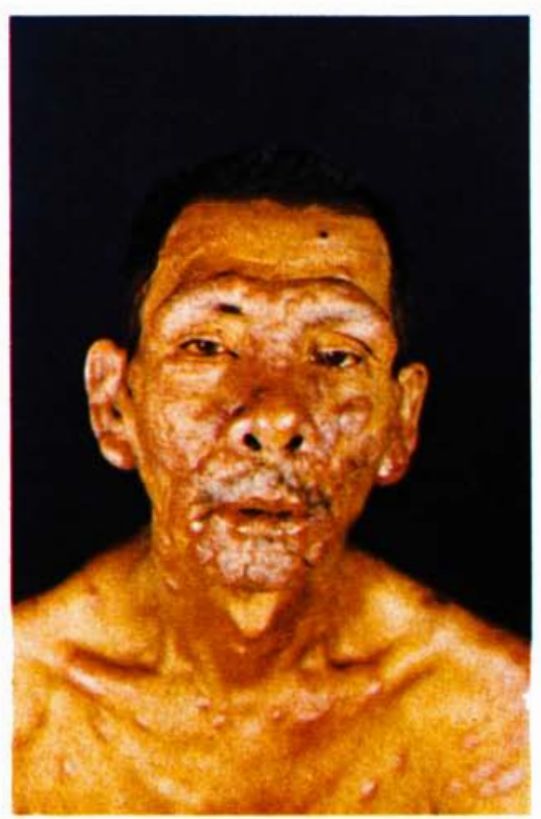

(b)

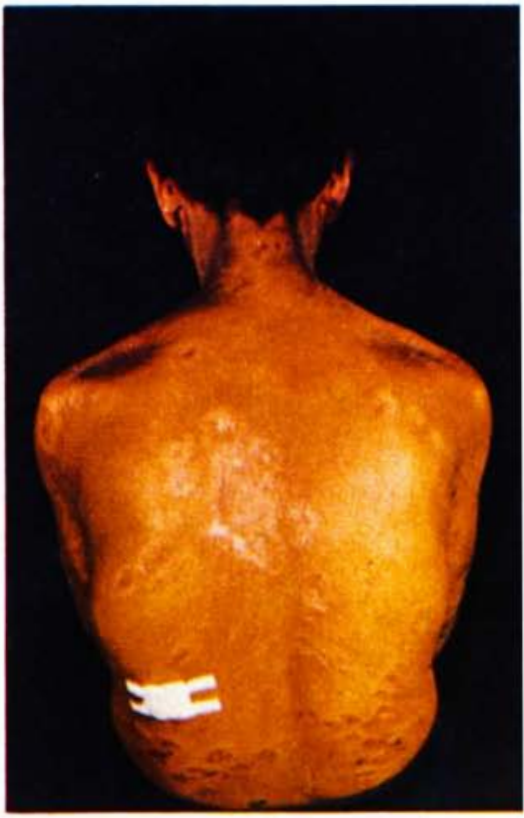

(c)

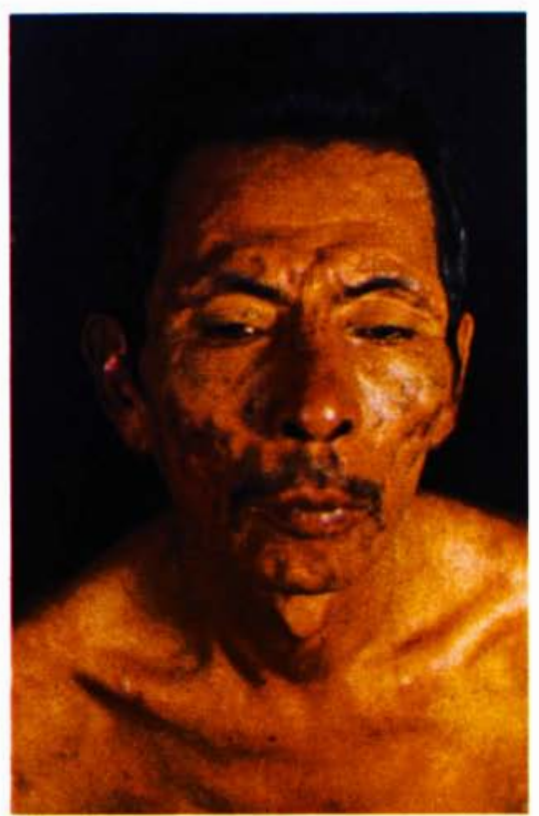

(d) 
infiltrations. The infiltration on the body disappeared fast leaving indistinct macules; infiltration on the face became thinner although it took a little more time becoming pink coloured but lighter than the initial red colour.

(7) G. T., age 43. Has completed 4 times follow-up. This patient underwent a gallstone operation 1967 and prior to this he was admitted to this hospital for 3 months because of epigastric pain. During the first weeks of the treatment, he complained of nausea, but later on this complaint disappeared without special treatment. After the 3rd follow-up, the patient was suffering from a serious illness with high fever accompanied by vomiting and diarrhoea. The general condition got worse, his weight dropped by $8 \mathrm{~kg}$ to $49 \mathrm{~kg}$. During his serious illness, the lesions on the skin did not increase although leprosy treatment was stopped for the time being.

\section{Lepra Reaction}

A mild leprosy reaction occurred in 3 patients but disappeared without special medication so that treatment could be continued without interruption. Details are as follows.

(1) A. A., age 60. From the 1 st month onwards some nodules occurred but appeared and vanished, mainly on the extensor part of the arm, sometimes on the palm of the hand and on the lower leg.

(2) S. K., age 11. At end of the 3rd month after having completed the 6th follow-up, some nodules appeared on both underarms and on the extensor part of the leg as well as on the chest.

(3) S. L., age 32. At the 1 st follow-up, 3 new nodules were seen on the extensor part of the left arm and 2 appeared on the palm of the hand. They disappeared slowly and no further new nodules occurred.

\section{Side-effects}

The side-effects found were nausea, vomiting, dizziness, weakness and insomnia, all of which disappeared without special treatment, except that 1 dropped out because of severe vomiting as reported above.

\section{Bacteriological Findings}

Out of the 8 patients who have completed 6 months of follow-up, 3 patients were still bacteriologically positive, viz.:

(1) Z. A., age 30, with several globi in the earlobes and solid forms of bacilli still present.

(2) M. T., age 30, with some globi in the earlobes.

(3) S. K., age 11, still with solid bacilli at the 6th follow-up but no globus. Other patients:

(4) M. M., age 31, negative for bacilli at the 6th follow-up

(5) M. A., age 50, negative for bacilli at the 6th follow-up

(6) A. L., age 65 , negative for bacilli at the 6th follow-up

(7) M. R., age 37, negative for bacilli at the 5 th follow-up

(8) W. A., age 53, negative for bacilli at the 3rd follow-up. 
Eight patients who had not reached the 6th follow-up gave the following results:

(1) A. N., age 60, negative for bacilli at the 4 th follow-up

(2) G. T., age 43, negative for bacilli at the 5 th follow-up

(3) S. L., age 32, negative for bacilli at the 4th follow-up

(4) A. G., age 42, negative for bacilli at the 1st follow-up

(5) A. B., age 31 , negative for bacilli at the 1st follow-up.

All these 13 patients started with globi in their smears except the 2 last cases.

\section{Histopathological Results}

Histopathological examination during treatment revealed involution of leproma with reduction of the infiltration, bacilli became granular and fragmented, and disappeared with the presence of fibrosis. The presence or absence of bacilli in the histopathological specimens was in conformity with the smear results.

\section{Laboratory Results}

There are no significant changes in the haemoglobin, leucocyte count, differential count, thrombocyte, SGOT and SGPT during the treatment. The initial high value of ESR gradually decreased during treatment.

\section{Discussion}

Compared with the treatment with dapsone as "drug of choice", consisting of a maintenance dose of $350 \mathrm{mg}$ per week in accordance with the regulation of the 10 th International Congress of Leprosy, the treatment which we are here describing could be regarded as a "bulldozer" treatment, considering the doses, combination of drugs and price.

Rifampicin $600 \mathrm{mg}$ per day is a costly treatment involving a price which either cannot be paid by most of the patients, or only with difficulty. However, if the results are satisfactory, it will still be worth-while.

The treatment of patients with the lepromatous type is considered by some to be a life-long treatment. We know that the lepromatous type is a contagious type. Life-long treatment and contagiousness are the two main factors stimulating research with the "bulldozer" treatment with the aim of shortening the duration of treatment and hastening the process of conversion to the non-infectious conditions, while at the same time reducing emergence of lepra reaction and possibility of sequelae consisting of diverse forms of deformity.

Compared with other drugs this treatment is able to heal the lesions of nodular lesions of miliary and lenticular size and in the healing of the infiltration in the ear and the face it seemed that a longer time of treatment was needed.

A distinct healing with this drug was obtained with the patient A. G., 42 years old, who had been treated with other drugs approximately five years without success.

On the contrary there were 2 patients in whom globus, granular/fragmented as well as solid forms of Hansen's bacillus were still found.

Lepra reaction was slight so that treatment with a specific drug was not needed.

Concerning side-effects such as nausea, vomiting and dizziness, such symptoms are understandable considering the high doses given once daily. 
The provisional conclusion of this research was that a longer time was needed to evaluate the efficiency of this kind of treatment.

\section{Acknowledgement}

We are very much indebted to Prof. Freerksen, Director of the Forschungsinstitut Borstel and his staff for their excellent assistance and for the availability of the drugs used in this investigation.

\section{References}

Cochrane, R. G. and Davey, T. F. (1964). Leprosy in Theory and Practice, 2 nd ed. Baltimore: The Williams and Wilkins Company.

Leiker. D. L. (1971). Chemotherapy of Leprosy. Int. J. Lepr. 39, 462-466.

Opromolla and Souza Lima (1965). Congresso International de Leprologia-Rio de Janeiro.

Rees, R. J. W. and Pearson, J. M. H. (1970). Experimental and clinical studies of rifampicin in treatment of leprosy. (Preliminary communications). Br. med. J. 1, 89-92.

Shepard, C. C. (1971). The first decade in experimental leprosy. Bull. Wld Hlth Org. 44, $821-827$.

Wilkinson, F. F., Gaco, J. and Stantabaya, E. (1971). Therapy of leprosy with rifampicin. Int. J. Lepr. 40, 53-57. 